CTASS: an intelligent framework for personalized travel behaviour advice to cardiac patients

Peer-reviewed author version

BATOOL, Tooba; VANROMPAY, Yves; NEVEN, An; JANSSENS, Davy \& WETS, Geert (2018) CTASS: an intelligent framework for personalized travel behaviour advice to cardiac patients. In: Journal of Ambient Intelligence and Humanized Computing, 10(12), p. 4693-4705..

DOI: $10.1007 / \mathrm{s} 12652-018-0847-7$

Handle: http://hdl.handle.net/1942/26213 


\title{
CTASS: an intelligent framework for personalized travel behaviour advice to cardiac patients
}

\author{
Tooba Batool $^{\mathrm{a}^{*}, \text { Yves Vanrompay }^{\mathrm{a}}, \text { An Neven }}{ }^{\mathrm{a}}$, Davy Janssens ${ }^{\mathrm{a}}$, Geerts Wets ${ }^{\mathrm{a}}$ \\ ${ }^{a}$ UHasselt - Hasselt University, Transportation Research Institute (IMOB), Agoralaan, 3590 Diepenbeek, Belgium
}

\begin{abstract}
Current cardiac rehabilitation programs intending to increase physical activity of patients suffer from a lack of knowledge about effective patient's activity profiles and their associated behavior. This leads to the fact that therapies are not completely tailored to the patient, causing non-adherence to the proposed treatment schedule. An important potential for increasing the physical activity level of patients is available in their daily travel behaviour that can be made more active. To validate this potential, we propose a Cardiac Travel Advice Support System (CTASS) digital framework for personalized travel behaviour advice to cardiac patients. The travel behaviour of the group of patients whose actual physical activity level is expected to be too low is monitored by a smartphone application that objectifies their daily activity schedules. The data from the schedules is analysed semi-automatically by the CTASS. Based on this analysis, the doctor can provide a treatment that is personalized to the specific contexts of the patient. In this way, we try to optimize their travel-related physical activity. Moreover, we predict the risk of non-adherence to the therapy taking into account the derived characteristics of the patient.
\end{abstract}

Keywords: Active travel behaviour, smart cardiovascular rehabilitation, physical activity level, behaviour prediction

* Tooba Batool. Tel.: +32 (0) 112691 11; fax: +32 (0) 11269199.

E-mail address: tooba.batool@uhasselt.be 


\section{Introduction}

Cardiovascular diseases (CVDs) are considered the principal cause of the majority of global deaths. 17.5 million people die due to CVDs annually, which constitutes $31 \%$ of the global deaths (2016a). CVDs are the set of diseases which are caused by the ailment in blood vessels and the heart itself. Risk factors of CVDs include obesity, physical inactivity, hypertension, dyslipidemia, alcohol, smoking cigarettes, social exclusion and aggression. Among the other risk factors of CVDs, physical inactivity is an important factor. Physical inactivity has caused 3.2 million global deaths among the non-communicable disease patients, of which CVDs are considered a subset (2016b).

A recent study focusing on behavioural risk factors for CVDs identified physical inactivity as a significant behavioural risk factor for CVDs (Vassilaki et al. 2015). CVDs are controlled and treated by "cardiac rehabilitation" (CR) programs, which can help reducing morbidity and mortality caused by the disease. Cardiac patients need long term care after they had a cardiac event such as stroke or heart failure. A multidisciplinary rehabilitation program is introduced to improve the clinical and behavioural life of the patients to lead a better quality of life. These CR programs target the risk factors of CVDs in patients. An element that strongly influences the success of these CRs is the extent to which the patient continues the agreed upon mode of treatment, especially in the case of limited supervision. Therefore, non-adherence to the cardiovascular therapy is a serious contributor towards increased treatment costs and poor health prospects (Rehabilitation 2004).

The importance of physical activity (PA) in primary and secondary prevention of CVDs demands to achieve a certain patient-specific level of active lifestyle. Physical activity for CVD patients is not necessarily limited to exercise and training sessions, but also refers to part of their daily routine work such as mowing grass, climbing stairs or bicycling, which are also called Leisure Time Physical Activity (LTPA). LTPA refers to discretionary time allotted to an activity which increases the energy expenditure (Bouchard et al. 2012). The patients are recommended to attend CR programs regularly, however attendance still varies between $21 \%$ to $75 \%$ internationally (De Vos et al. 2013). CR programs mainly focus on dedicated exercise and training sessions, while patients remain sedentary in their other life routines. Persistent sedentary lifestyles in chronic patients have encouraged doctors to look at physical activity opportunities from a broader perspective. A potential for achieving a more active lifestyle can be found in the day-today displacements that people make and specifically in the associated travel modes of the trips. Moreover, travel behaviour data is not only useful to motivate people into active travel modes but also provides a unique opportunity of getting a deeper insight into the patient's sedentary lifestyle. This provides a treatment tailored and contextualized to the patient. To monitor, analyse and present the patient's daily routine (contexts like which trip they carried out at what time of the day and the duration), we propose a semi-automatic framework which will be able to support the key actors (patients and doctors) in achieving the travel-related active lifestyle.

Routine travel behaviour data generally includes six dimensions: 1) where? (location) 2) what for? (activity type), 3) when? (start and end time), 4) how? (transport mode), 5) with whom? (travel partner), and 6) for how long? (duration) (2009). The use of travel partner information has been proven that social interactions play an important role in mode choice and travel behaviour patterns (Kroesen 2015). These six dimensions constitute the daily schedules of an individual. The combination of this information along with other contextual information (Neven 2015) (e.g. demographics, attitudes, norms and perceived behaviour) provides a rational understanding for sedentary routine in travel behaviour. This information can be used by the doctor to advice the patient to change his lifestyle.

The purpose of this research is to provide a smart CVD rehabilitation framework for increased active travel-related physical activity in the patients. For this purpose the daily schedules of a patient will first be obtained in a way that will not burden the user through using smartphone applications (data collection). The data will be processed in a semiautomatic advice support system which will help the doctors to prescribe a contextualized and personalized physical activity scheme taking into account the travel behaviour of the patients. In case of non-adherence to the prescribed therapy, the system will allow to detect the deviation from the prescribed and agreed pattern. The travel data will be gathered by a smartphone apps, aggregated, analysed and visualized to the doctor by means of a web-based dashboard which is part of the Cardiac Travel Advice Support System (CTASS). In addition to detection of poor adherence by monitoring patients behaviour we also provide machine learning models that 1) predict the likelihood that a specific patient's physical activity level is too low, and 2) predict which patients have a high risk of not adhering to the prescribed therapy.

This paper is organized as follows. Section 2 gives an overview of related work, after which section 3 describes the different steps in the patient-doctor flow. Section 4 details the components and architecture of the framework, after which we conclude and discuss future work. 


\section{Related work}

An integral part of CR programs is the assessment of the physical activity of patients. In the past this was mainly done by patient's self-reporting through questionnaires such as the International Physical Activity Questionnaires (IPAQs) (Hagströmer et al. 2006), Global Physical Activity Questionnaire (GPAQ)(Cleland et al. 2014) and Physical Activity Questionnaire for Older Children (PAQ-C) (Pr et al. 1997). Physical activity level is also monitored by motion sensors such as accelerometers, which provide more objective measurements than questionnaires. However, such technologies also have some limitations. The patients usually have to place the accelerometers on a proper position (hip) leading that it cannot distinguish between sitting and standing still and neither they are able to give information on body posture (Lee and Shiroma 2014). More importantly, they provide only basic metrics without any contexts such as duration, time of the day, with who and which kind of activities they are performing.

Recently, smartphone apps which require minimal user intervention to provide rich information on patient physical activity (Ham et al. 2016), have increased their use in cardiology. Smartphone-based cardiac health provision is delivered in two ways: 1) outpatient and inpatient continuous multi-parametric sign monitoring, and 2) prevention of cardiac events through risk factor management apps (weight management, physical inactivity, blood glucose control, smoking cessation) (Nguyen and Silva 2016). Studies have proved the positive role of smartphone technologies in increasing the adherence of the patient to the proposed therapy and in providing more cost effective solutions. A meta-analysis by Park et al. concluded that text messaging, mobile applications, and tele monitoring via mobile phones effectively improved prevention and management of cardiovascular diseases. These studies also stressed the importance of personalized and tailored advice to maximally target CVD risk factors (Widmer et al. 2015; Park et al. 2016).

Smart homes and ambient assisted living (AAL) technologies leverage infrastructure and machine learning techniques for monitoring living spaces, modeling and recognizing activities (Amiribesheli et al. 2015). While AAL is hugely promising, its adoption rate until now is rather low (Calvaresi et al. 2017). Indeed, exploiting ICT-solutions in capturing, analyzing and promoting physical activity offers substantial improvements in physical activity level. A recent meta-analysis concluded that patients who follow advice and support material on social media are more active. Smartphone apps and self-monitoring devices such as pedometers succeed in providing the means for feedback to the patient in the form of situation-tailored advice to improve the physical activity level (Chaddha et al. 2017). Examples range from rather complex fitness gadgets such as Fitbit, Garmin, Mio Global and Samsung Gear Fit2 to smart phone apps such as RunKeep and Strava Running. Additionally, paper-pen travel diaries are proved to adversely affect the data quality due to under-reporting of participants and tend to have a high cost. A recent study by Safi et. al compared four technologies (web-based, H-tracker, S-tracker and ATLAS-II data collection methods) for travel surveys and concluded that smartphones provide better accuracy of data (Safi et al. 2017). Many sophisticated systems have been developed so far to semi-automatically detect and record digital travel diaries, including FMS (Future Mobility Sensing), MoveSmarter, SmartMo (Berger and Platzer 2015; Geurs et al. 2015; Zhao et al. 2015) and SPARROWS (Smart Phone App for Remote Registration Of WhereaboutS) developed by IMOB (UHasselt 2012). Health apps (e.g. Moves, journeys, ForGood) related to physical activity provide the information on most of the parameters (location, timing, duration and distance) of travel behaviour. In our study, the remaining parameters (transport mode, activity type and travel partner) are obtained by Health in Travel Behaviour (HTB), a Moves-connected app, described in section 3.1. Travel diary systems like FMS, MoveSmarter and Sparrow use web-based recall survey diaries which require explicit and rather elaborate input from the users. SmartMo records trips of a user but requires to manually start and stop a trip and provide trip information. we have tested and compared a few smartphone apps with similar functionalities such as Journeys, ForGood and Moves. Journeys and ForGood are smart applications based on learning mechanisms. They automatically identify the transport modes and the type of activities of its user. The researcher has tested these applications for 3 months. However, automatic detection is not yet accurate, as for example both applications (Journeys and ForGood) cannot differentiate between motorized transport such as car and bus. Similarly, the activity type recognition is also not accurate. The algorithm of the application tends to learn with time and gives much better results after time but these are still not accurate enough. Another reason for not selecting these applications, was the level of difficulty regarding its user interface. On the other hand, Moves is a free source application which provides rather accurate information and easily available API to connect and export the data. 
Completely accurate automatic recognition of transport modes and activity type is not possible yet; but the Moves app proved to be the best possible option regarding data accuracy, data accessibility and user interface.

Therefore, we propose a novel integrated solution for objectively measuring the daily activities of patients and adding the needed travel related parameters using smartphone apps in a way that requires minimal user input.

Until now, the integration of health and mobility has been rather limited in exploring the health impacts of active transport modes. Existing studies have focused on the following areas in integrating health and mobility: 1) policyrelated measures like transportation goals and investments, 2) improving the built environment, e.g. road networks and accessibility (de Nazelle et al. 2011), and 3) economic values, e.g. the health cost of motorized transport (Newman and Matan 2012).

The fact remains that studies linking epidemiology and travel behaviour lack in exploring complex causal relationships of contextual factors of patient lifestyles (day-to-day travel pattern/daily schedules). This contextual information provides insight into the less active lifestyles. In this way patients can be motivated to be more active in their lifestyle by agreeing on the advice recommended by the doctor. In this research, we investigate the potential of integration of physical activity (which is objectively measured through an app) as a health factor and six dimensions of travel behavior in order to make patients more active in their day-today displacements. The integration of sociodemographic information, personal attitudes, norms and perceived health and travel behaviour data is obtained through a framework which works as a digital cardiac physical health care advice assistant. This system will help in understanding the role of travel behaviour parameters in increasing the physical activity level of patients and realizing a flexible, patient-specific advice support system for doctors. The integration of travel behaviour in CR program using a semi-automatic framework is a new perspective that potentially benefits achieving a certain level of PA.

\section{Methodology}

The CTASS framework described in section 4 will be integrated in the experiment which consists of the following stages:

1. After getting the approval from the ethics committee, we will recruit 500 Coronary Artery Disease (CAD) patients from the cardiology centre of our partner hospital. we will obtain their demographic information, baseline health parameters, attitudes, norms, perceived behaviour and household information through an online survey. The data from this questionnaire will be saved in the data repository as explained in section 4.3 .

2. Travel behaviour monitoring of the patients with will be carried out for at least 3 weeks for the first period by using smartphone apps Moves and HTB (retrieving data on six travel behaviour parameters). Patients have to annotate their information on the smartphone app on a daily basis. The smartphone app will send notifications each two days if there are some missing values present in the data. The data will be uploaded automatically to the server on regular time intervals. The patient identities are coded and the information retrieved from the smartphone applications will be anonymised for research purposes, but the doctor will be able to identify the patients.

3. After the data is stored on the server, analysis and generation of the reports will start. The system will know the patients identity and will combine the survey information of that patient with the smartphone data. By combining survey data and travel parameters, the automatic reasoner aggregates the data, learns the heuristics and generates the initial reports. The initial reports will assess the baseline physical activity level in travel behaviour. The patients will be able to visualize this on their smartphone screens and the doctors will see the reports on the web- based dashboard management system.

4. After the patients will receive the reports, an interactive feedback session between patients and doctor will be held. The doctor (physiotherapist) will discuss the aggregated physical activity level in travel behaviour, leading to contextual and customized advice taking into account different scenarios with patients.

5. After the interactive session (recommendation of advice), the patient will be asked to monitor his travel behaviour again for a second period (for 3 weeks at least). Monitoring of travel behaviour for the first period, the interactive session and monitoring for the second period will take place simultaneously during the CR program which continues for 3 months. It will be complementary for the patients to continuously monitor the travel behaviour for the duration of the CR program (3 months).

6. The CTASS framework will perform a comparative analysis on the data before and after the interactive 
session to observe the resulting changes in patient's lifestyle.

7. The patients will be advised to continue monitoring for a minimum 6 month period so that this data can be used for the development of the automatic advice reasoner. This reasoner is based on contextual information and neural network models, which leads to the generation of advice based on contextual parametric information. This advice takes into account the expected physical activity level of a patient and the likelihood of that patient adhering to the prescribed therapy.

To summarize the current state of the research project, the technical components of the CTASS framework are in place (online questionnaires, HTB app, Cardiac Travel Advice Support Service Architecture, Intelligent Health Reasoner Prediction Component and dashboard). We will start experimental study by getting the approval by the appropriate ethical committees and recruiting patients for collecting their data.

\section{Components of the CTASS framework}

This section provides an overview of the components in the CTASS framework. First we describe the data collection components of the system:, i.e. online questionnaires and the HTB app. Then we present the overall architecture of CTASS, after which we zoom in to the Intelligent Health Reasoner Service as a central component. Finally, the web-based dashboard that visualizes all patient information for the medical personnel, is explained.

\subsection{Data collection Components}

\subsubsection{Online questionnaires}

Baseline information of patients will be asked from the patients through the online surveys that are conducted while recruiting and after recruiting in the preparatory group session. An online survey will be conducted through a website to check the inclusion and exclusion criteria for the study; which consists of some medical parameters such as weight, height, number of admission in the hospital, etc. After the recruiting survey, the patients will be invited for a preparatory session, which will be held to provide information and to instruct how to use the smartphone applications Moves and HTB. The patients fill in an online questionnaire which consists of the three following blocks:

1. Personal information: age, educational level, gender, personal income and profession, .... .

2. Household information: net household income, number of household members and their details, ... .

3. Information related to travel behaviour: total number of vehicles in household, distance to near public transport stations, behaviour and attitude towards use of active transport modes.

The information collected through these online surveys will be used to determine the correlation between personal profiles and daily monitoring of travel behaviour. It is also used to contextualize the gathered data and to derive the features needed as input for the prediction models.

\subsubsection{Health in Travel Behaviour app (HTB)}

The Health in Travel Behaviour (HTB) app is developed for the study to connect to the physical activity app (Moves) to obtain the complete overview of a patient's travel behaviour as mentioned in section 2. Parameters required in the app for travel diaries are based on National Household Travel Survey (NHTS) 2009, United states and Onderzoek VerplaatsingsGedrag (OVG) Vlaanderen, Travel Behaviour Research Flanders, Belgium.. Moves, an existing third-party app, is leveraged as a state-of-the-art activity tracking system which provides information on activity and trip duration, activity location and transport modes. Moves can provide information on walking, cycling and motorised transport modes. However, for a motorised transport mode, the user needs to manually enter the exact information (car, bus or motorbike etc.). Besides, information on parameters such as travel partner and activity purpose cannot be retrieved from Moves directly. Therefore, the HTB app collects the information of patients on transport mode, activity type and travel partner. Both the Moves and the HTB app are available on android and IOS platforms.

Manual annotation of the exact transport mode in Moves is not needed for the study, as this information is obtained through the HTB app, along with the other two parameters activity type and travel partner. The interaction between the HTB app and the Moves is explained in Figure 1.

1. The user logs in to the HTB app by calling the HTB API.

2. The HTB app calls on the HTB API to get Moves data, to be annotated by the user. 
3. If the user does not already have a Moves token that allows him to access the Moves API, the HTB API gives an error 460, after which the HTB app needs to authenticate itself with a user id and token, by being redirected to the Moves app. As a result, it gets an authorization code form the Moves API.

4. Finally, the HTB backend receives this authorization code, and can access the Moves API to get the data, and give it back as a result to the HTB app "Get Moves data" call.

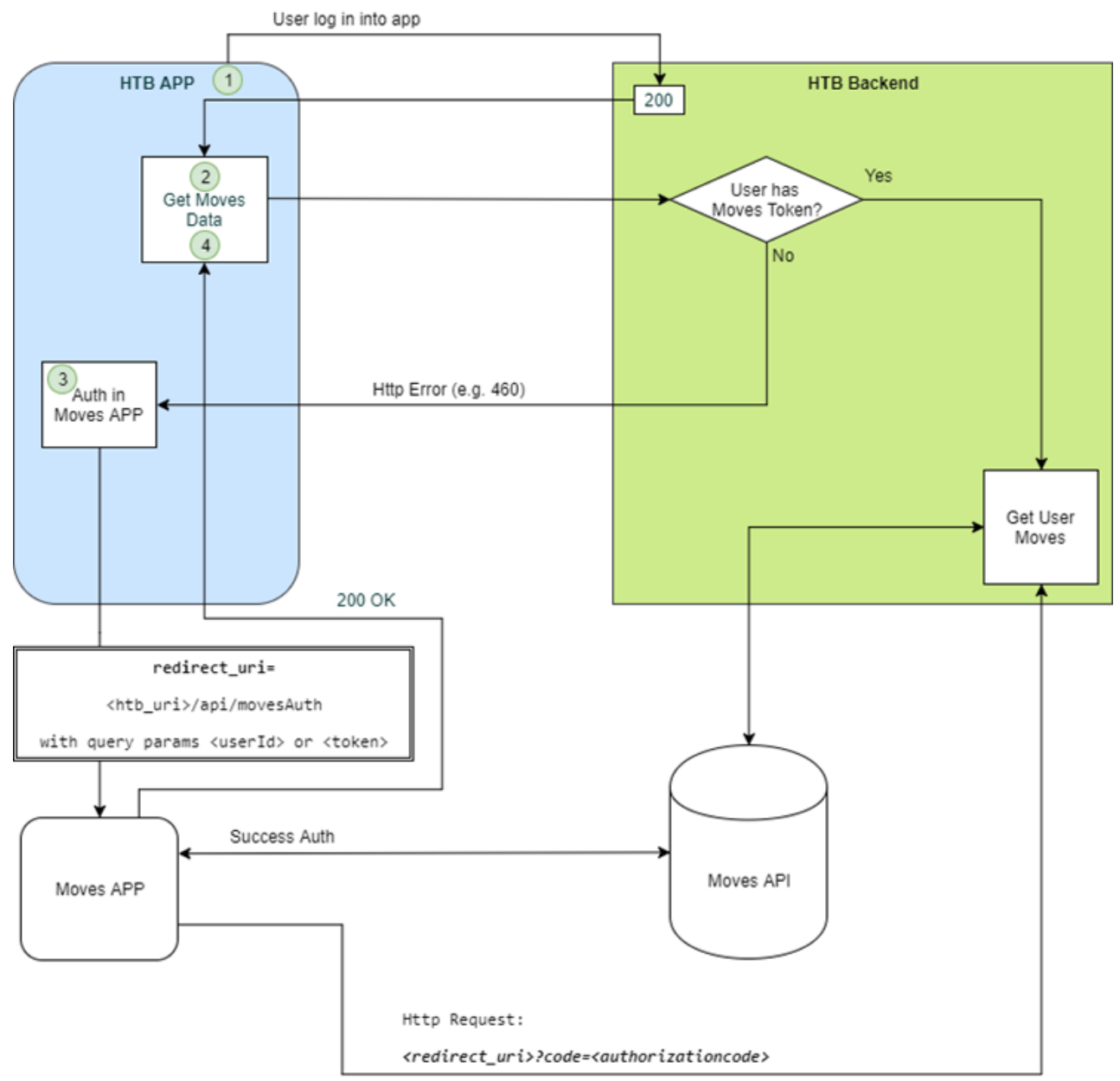

Fig. 1 Interaction between the Moves and HTB apps

To summarize, data is received by the HTB app by calling the HTB API, which then calls the Moves API to get the data. If needed, by exchanging a token and a user id, from the HTB app (by redirection to the Moves app), the required authorization code is obtained. Both the Moves app and the HTB app run independently from each other on the same smartphone, and interaction is limited to delegating authorization to the Moves API by communication between the HTB and the Moves app. The data pull itself is done by the HTB backend calling the Moves backend.

The HTB app together with Moves forms a complete digital travel diary that provides detailed (objectively measured) information, using no custom device except for a smartphone. There is no need of web-based recall surveys and we include detailed measurements of all transport modes in a trip segment. This information is important for the study to keep track of physical activity levels achieved during the travel plan of the patient. The information is then sent to the data repository of the CTASS framework for processing. Figure 2 illustrates how a user annotates the parameters in the HTB app. Screen $a$ presents the sequence of the activities during the day after annotation as shown to a user. Screen $b$ presents the list of activities (i.e. home, business and work etc.) that can be associated with a 
location. The user has to choose the relevant activity by tapping on the location label. Screen $c$ shows the attributes with their possible values which the user can select while annotating his trips: transport modes and travel partner.

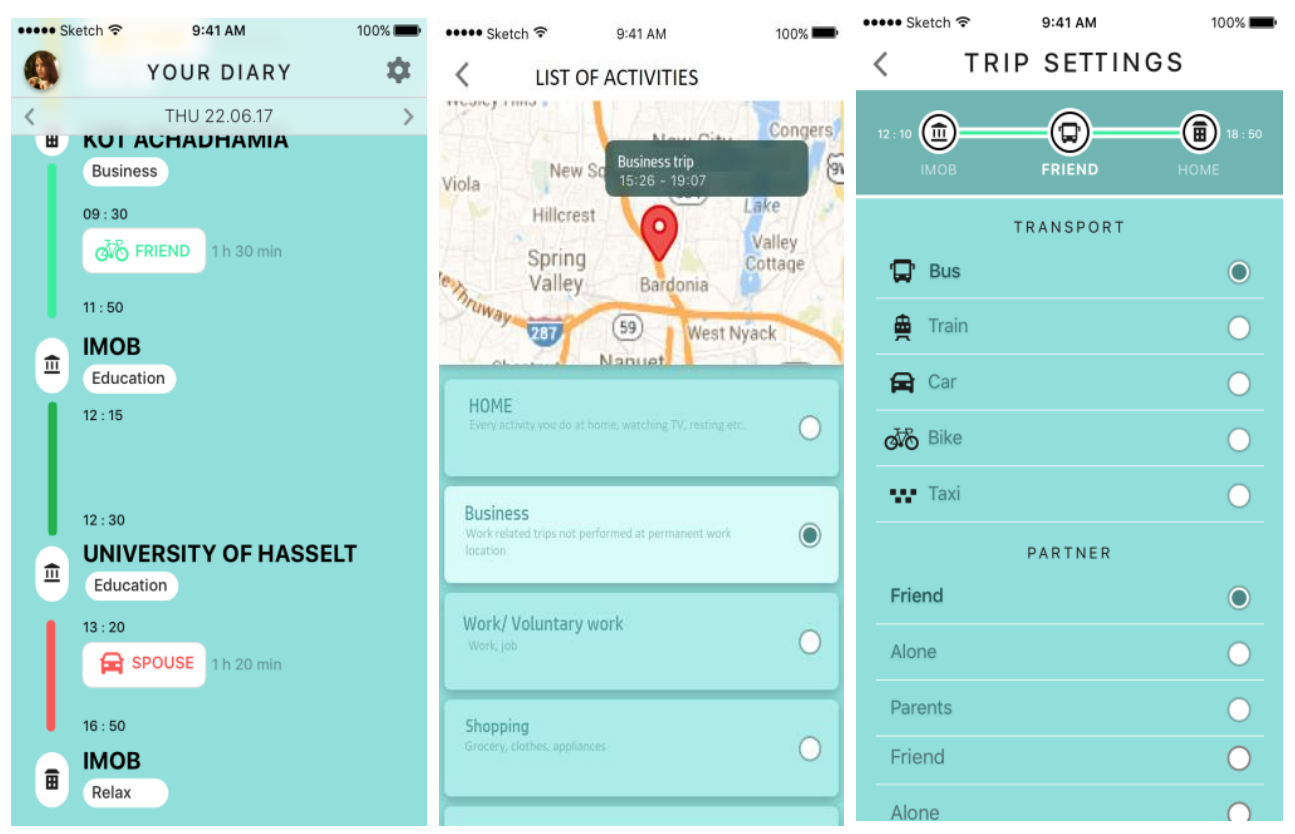

Fig. 2 Basic functionalities of the HTB app

\subsection{Cardiac Travel Advice Support Service Architecture}

This section gives an overview of the architecture of the Cardiac Travel Advice Support Service (CTASS). The framework is developed to be open, generic, pluggable, easily extendable and configurable. The framework is generic in the sense that any app or wearable which provides an API from which data can be pulled can be plugged in and its data can be consumed. Preprocessing brings the data in the correct format for further analysis. Data providers can also specify their data scheme and sensor dependencies in XML such that the correct sensors can be activated when needed. A variety of learning algorithms, statistics and data representation techniques in the intelligent reasoner component act as a library for handling heterogeneous data.

As can be seen in Figure 3, an API is provided which allows for third-party apps to connect to the system and to act as information providers. The CTASS can also itself connect to the API of external branded health parameter sensors to get health data from devices like blood pressure meters and motion sensors. Typically a brand will push a notification to the CTASS ("Notification Listener") that new data is available, after which the data is pulled by calling their API. We have incorporated a pull model: data is pulled by the CTASS server component from the Moves API once each 24 hours (at night) for the previous day and stored on our server. The Moves API calls return a LastModified header in the response with some timestamp in RFC 1123 format. We provide an If-Modified-Since header in the API call for optimization so that if the data for the date range has not been updated since then, the Moves server will respond with a 304 Not Modified response code and an empty body.

In ideal conditions, the Moves API provides at most a couple of hours old user data. User data is uploaded from the Moves app to the Moves servers whenever the user opens the app in his phone and also periodically every couple of hours. The data updates may be irregular if the user has bad internet connection or is using the Moves battery saving mode.

According to the Moves app website, the battery usage is optimized as far as possible, within limits of what is possible. To improve the power consumption a nightly charging is recommended by the developers. A smartphone 
using the Moves app, with a typical phone use, can have enough battery to last all day. A day of typical phone use can last for 14 hours on an iPhone 4S and medium range android devices. There is an optional battery saving mode in the Moves app for iPhone which saves battery up to 40\% ("Moves - Activity Diary for iPhone and Android", https://moves-app.com). The HTB app does not use any phone sensor like Moves does to track location. It only gets data from the HTB API and sends data back to it. Since this data transfer is limited to json files, battery consumption of the HTB app is limited.

The HTB app (a Moves connected app, as described in the previous section), is one of the different health information providers plugged in to the CTASS. The HTB app will regularly post its collected data to the server, transparently for the user, when a known Wifi connection is available. The type of information provided by a plugin component is specified in XML together with a set of rules such that the "Data Pre-processor" and the "Intelligent health reasoner service" know what the data is and how to process it. The daily trip reports are pulled from the Moves API in the XML format specified by Moves (see XML snippet below). A daily trip is represented as an ordered set of segments, where a segment can be a place or a displacement (type: move). Activities are part of a displacement, containing type of activity, coordinates, travel partner, number of steps, and other relevant information. Since for our purposes, we need to annotate the data further, json keys for travel partner and more specific transportation modes are added to the XML on the HTB server before being sent to the HTB app for further annotation.

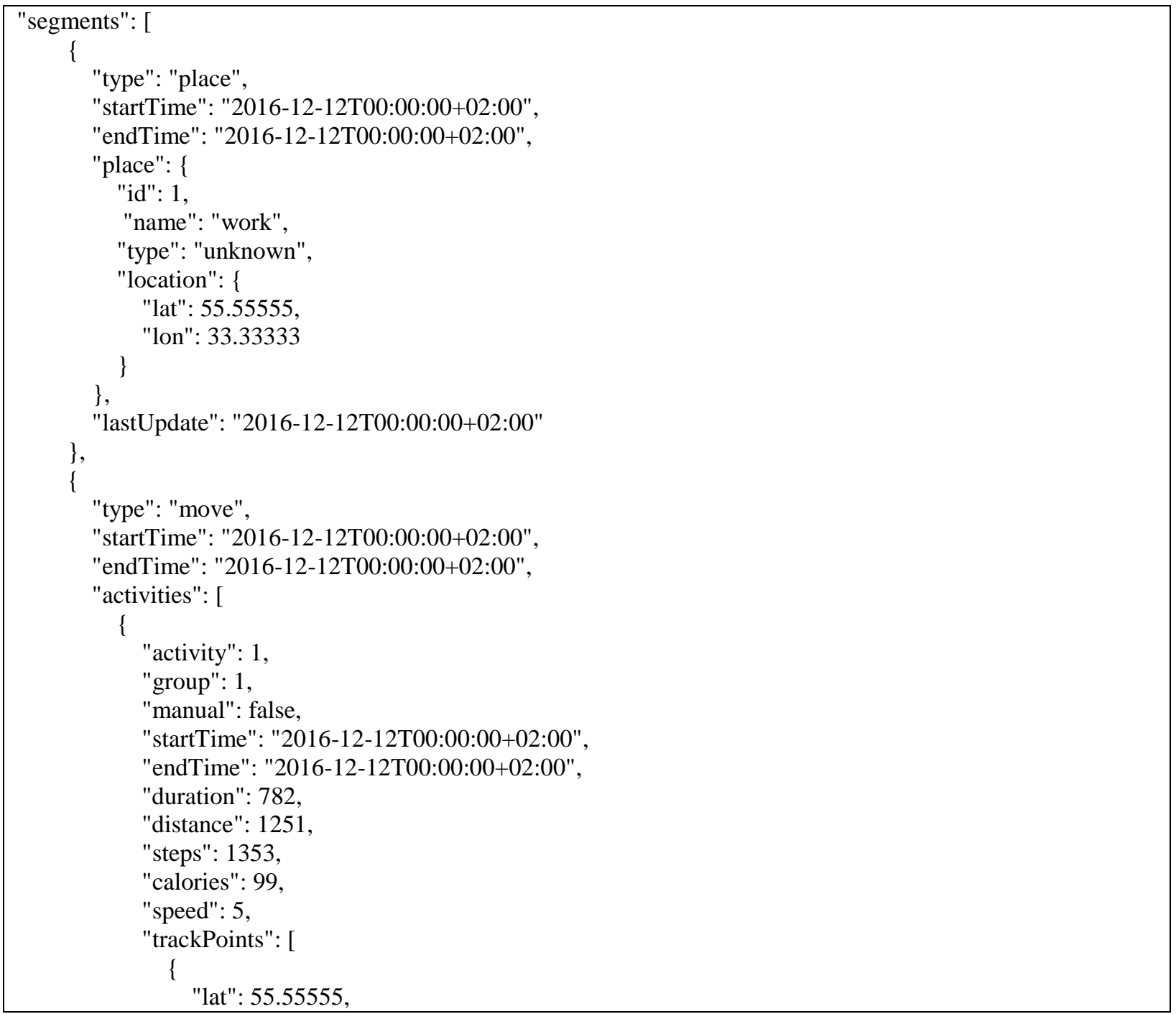




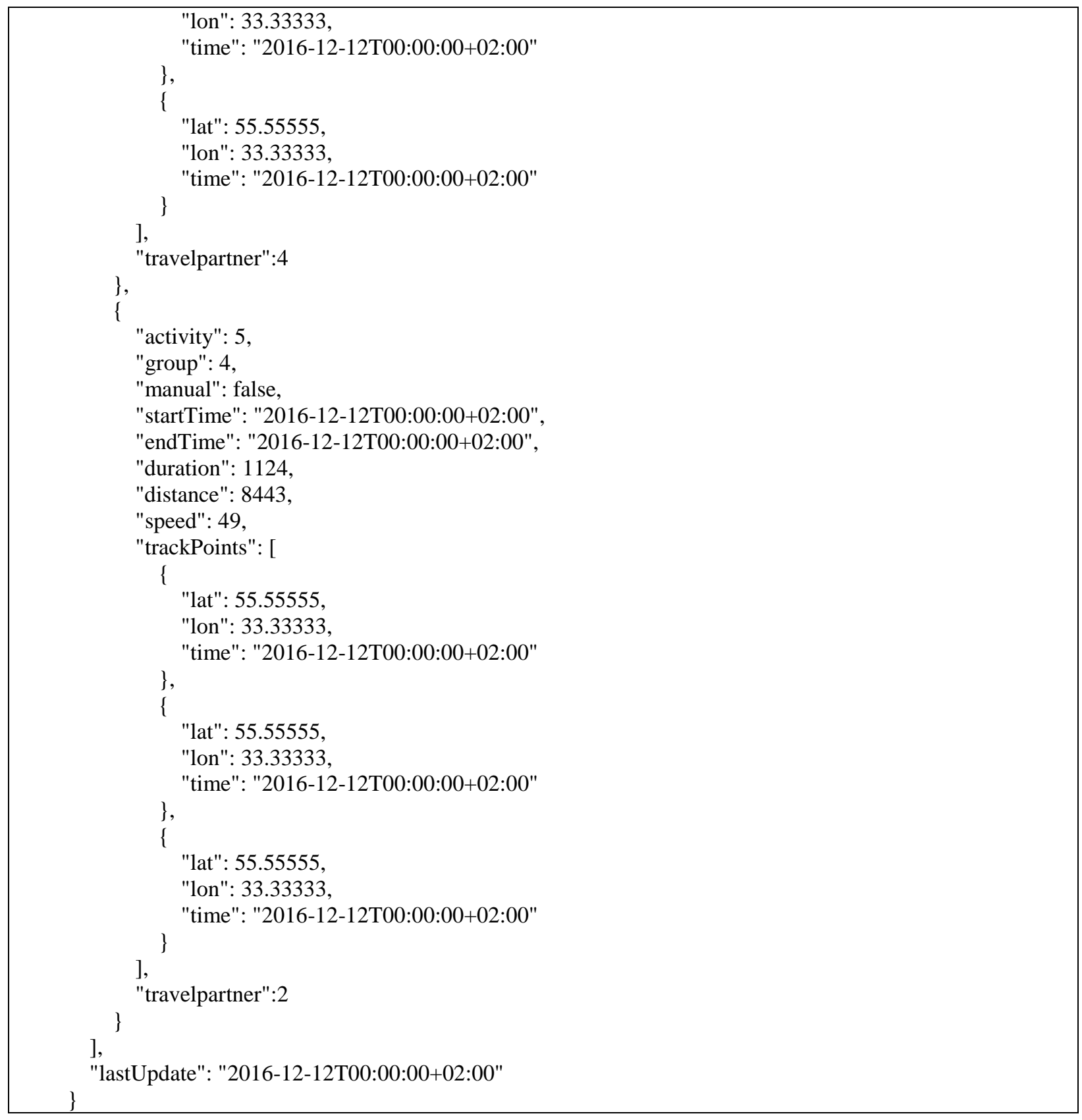

After the received patient data is pre-processed automatically to treat missing values by the data pre-processor component, it is stored in a database in a secure data repository. The database resides in a private cluster only accessible by the CTASS service, and personal data is scrambled such that measurement data cannot be linked to specific patients. Data is stored on a secure server in the cloud, with the AES-256 encrypted database in a virtual private cloud, which allows inbound traffic only by the SSH protocol from a fixed and known IP range. All other ports are closed. In the data schemas itself, there is no explicit link between patients and ids. This means that only the medical personal who has the link between the patient names and ids offline (on paper), can identify who the data belongs to. This repository also contains demographical data about the patients, health parameter specifications and patient contextual data which is obtained through the filled-in questionnaire. The "Intelligent health reasoner service" aggregates this data on a regular basis, produces statistics and adds contextual information to the statistics. The 
information of each patient (questionnaires answers and HTB data) is aggregated to generate reports on the daily trips with their properties. The aggregator also acts on the data to provide the features necessary for training and using the neural network prediction models, which are presented in the next section 4.3. These models are capable of 1) predicting the likely physical activity level of a patient based on the questionnaires answers, and 2) predicting the likelihood that a patient adheres to a prescribed therapy, based on the questionnaires answers and HTB data.

If a patient's health parameter is not within a (configurable) range, a warning is triggered by the "Alert Generator". This warning can trigger pushing a message to the patient's HTB app to ask the patient for the context in which the alert was received so that the doctor can assess its severity.

Aggregated information and statistics of each patient are presented to the doctor by means of a "Web-based visualization and management dashboard", which is explained in Section 4.4. This will allow the doctor to get an indepth insight into the physical parameters and travel behaviour of each patient, by which CTASS acts as a decision support and advice system to tailor and to adjust the treatment to the specific context of the patient. As will be explained in the next section, the doctor is also able to assess the current expected physical activity level of a patient as it is predicted by the patient. Moreover, the doctor is able to assess the predicted or expected risk of non-adherence to therapy for that particular patient.

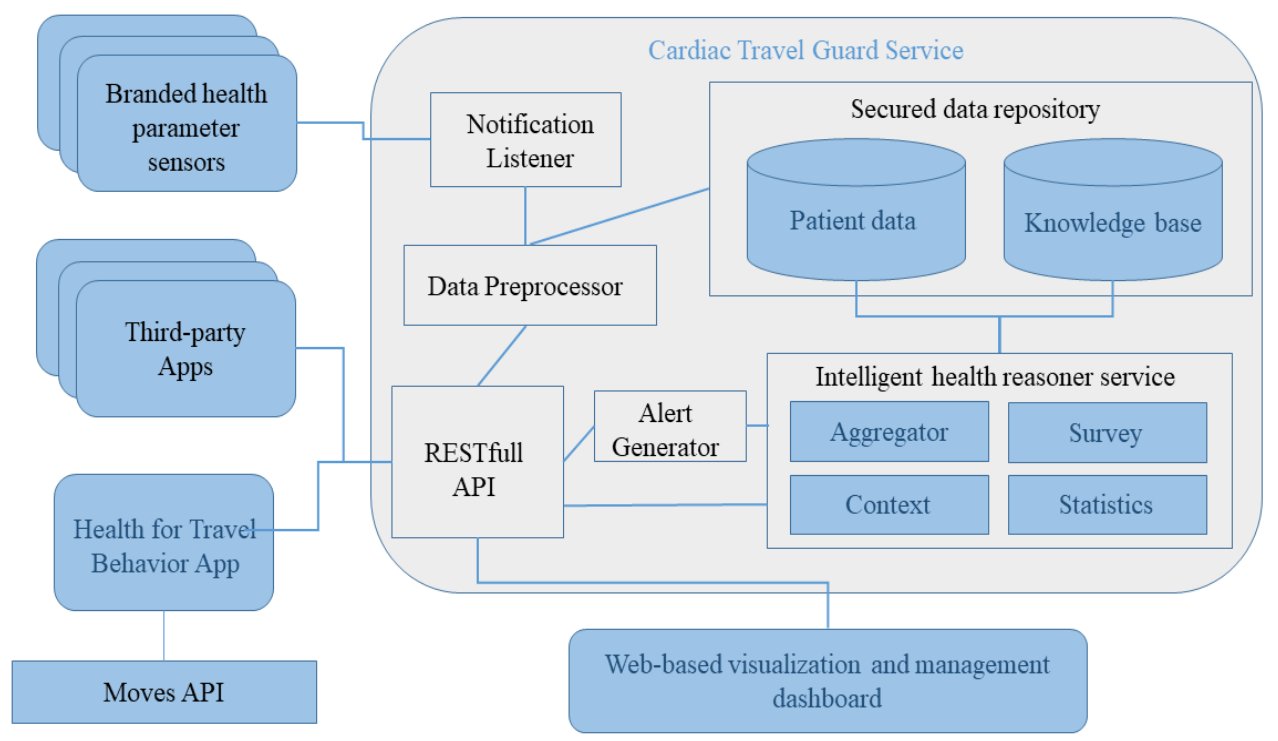

Fig. 3 CTASS framework architecture

\subsection{Intelligent Health Reasoner Prediction Component}

The intelligent health reasoner component pre-processes and aggregates the information obtained from the questionnaires and inputs these as features to neural network models. For a deep learning approach to work well, it is necessary to have a significant amount of training data available (i.e. Big Data). Given our study small group of cardiac patients, we had to balance the amount of training data and complexity of the learnt model. Therefore, a neural network with a hidden layer seems both feasible and sufficient for our approach. The dimensionality of the input space is relatively high for certain machine learning techniques. On the other hand, the amount of training data given the number of patients is relatively low, making a deep learning approach with a high number of hidden nodes unfeasible to train. The number of nodes in the hidden layer is chosen as best practice given the number of nodes in the input layer and the expected number of training examples. Neural network models are leveraged for two different purposes:

- Physical Activity Level Prediction (PALP) model: Based on the features derived from the user's answers to the questionnaires, the expected physical activity level of the patient is predicted. Note that the predicted physical activity level in our case only takes into account physical activity during daily trips. The expected physical activity allows the doctor to assess whether this patient probably needs extensive 
or only minor therapy advice to change his physical activity to the desired level. If the predicted physical activity is far below the desired level, the doctor can decide that the patient should monitor his actual physical activity using the HTB app.

- Prescribed Therapy Adherence Prediction (PTAP) model: Based on the user's answers to the questionnaires, the actual activity level as measured by the HTB app, and the desired activity level as prescribed by the doctor, this model predicts the risk of that patient not adhering to the prescribed therapy.

The features that are used as inputs for the neural network models are listed in Table 1.

\begin{tabular}{|l|l|l|}
\hline Type of feature & Feature & Values \\
\hline Demographic & Age & Number of years \\
\cline { 2 - 3 } & Gender & Male - Female \\
\cline { 2 - 3 } & Marital status & 5 categories \\
\cline { 2 - 3 } & Driving license & Yes - No \\
\cline { 2 - 3 } & Education level & 8 categories \\
\cline { 2 - 3 } & Professional activity & 3 categories + "None" \\
\cline { 2 - 3 } & Work schedule & 4 categories \\
\cline { 2 - 3 } & Net income & 5 categories \\
\cline { 2 - 3 } & Type of living area & City, Suburb, Rural \\
\cline { 2 - 3 } & Weight & Number of kilograms \\
\cline { 2 - 3 } & Duration of disease & Number of years \\
\cline { 2 - 3 } & Perceived capacity to walk & Duration (number of minutes) \\
\cline { 2 - 3 } & Perceived capacity to bike & Duration (number of minutes) \\
\cline { 2 - 3 } & Number of cars at home & Number \\
\cline { 2 - 3 } & Number of bikes at home & Number \\
\cline { 2 - 3 } & Distance to bus stop & 4 categories \\
\hline Intentional & Attitude & $\{1,2,3,4,5,6,7\}$ \\
\cline { 2 - 3 } & Subjective norms & $\{1,2,3,4,5,6,7\}$ \\
\cline { 2 - 3 } & Perceived behavioural control & $\{1,2,3,4,5,6,7\}$ \\
\hline Habitual & Habit & $\{1,2,3,4,5,6,7\}$ \\
\hline Activity level & Actual activity level & Float \\
\cline { 2 - 3 } & Desired activity level & Float \\
\hline & & \\
\hline
\end{tabular}

Table 1 Features of the prediction model

All features listed have numeric values or are transformed into numeric values. The features representing demographic data consist of a number (e.g. "number of cars", "age") as value, or are categories which are transformed into a number. For example, the values of "type of living area", being (City, Suburb, Rural) are mapped onto the numbers $\{1,2,3\}$.

The features "Attitude", "Subjective norms" and "Perceived behavioural control (PBC)" together form the set of intentional features (Francis et al. 2004). The attitude towards a behaviour is a person's overall evaluation of that behaviour. It contains beliefs about consequences of behaviour and corresponding positive or negative judgements about the behaviour outcome. Subjective norms are someone's feeling of the social pressure to behave or not behave in a certain way. The PBC expresses how well a person thinks to be able to perform a certain behaviour. In general, the more positive the attitude and subjective norms, and greater the perceived control, the stronger is the intention to perform the behaviour (Francis et al. 2004).

The values of these features are a number between 1 and 7 . These values are derived from the responses given by the patient to the relevant questions in the questionnaires. An example question that assesses the subjective norm of a patient regarding using active transportation is shown below: 


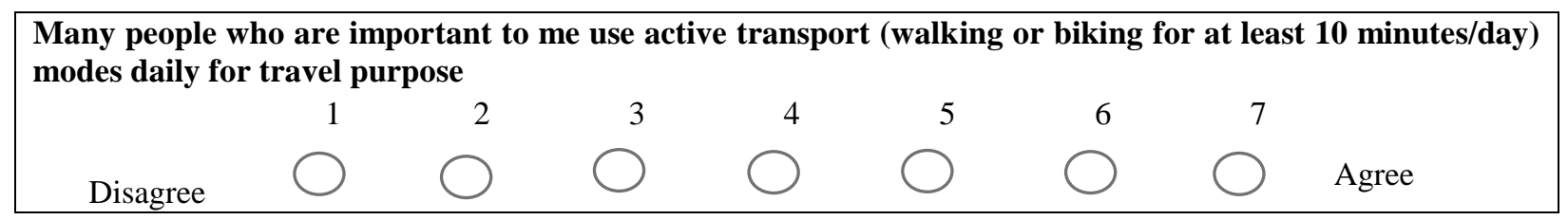

The feature "habit" corresponds to repeated behaviour which is consistent in a similar context for the same purpose. The frequency of past behaviour is generally acknowledged to play a significant role in the prediction of goal-directed behaviour (Danner et al. 2008). An example question assessing habits of a patient with respect to using active transportation is the following:

\begin{tabular}{|l|llllllll}
\hline \multicolumn{6}{l}{ I start using active transport (walking or biking) modes daily during my travel before I realize that I am } \\
using them \\
Disagree
\end{tabular}

The values of the intentional and habitual features are calculated using the rounded mean of the responses of the set of questions relevant for each feature. Two additional features are used in the PTAP model: actual and desired activity level. To estimate the actual physical activity level, we use the following equations from the literature (Gerrior, Juan, and Basiotis 2006; Thompson and Kayak 2011):

Calculating the Basal Energy Expenditure (BEE) for male:

$\mathrm{BEE}_{\text {male }}=293-3.8 \times$ age $($ years $)+456.4 \times$ height $($ meters $)+10.12 \times$ weight $(\mathrm{kg})$

Calculating the BEE for female:

$\mathrm{BEE}_{\text {female }}=2472.67 \times$ age $($ years $)+401.5 \times$ height $($ meters $)+8.6 \times$ weight $(\mathrm{kg})$

Calculating the physical activity impact on Energy Expenditure ( $\triangle \mathrm{PAL})$ :

$$
\Delta \mathrm{PAL}=\frac{(\text { METs }-1) \times[(1.15 / 0.9) \times \text { Duration }(\text { minutes })] / 1440)}{B E E /[0.0175 \times 1440 \times \text { weight }(\mathrm{kg})]}
$$

Calculating the Physical Activity Levels (PAL):

$$
\mathrm{PAL}=1.1+\Sigma \Delta \mathrm{PALi}
$$

Fig. 4 Estimation of physical activity level

The desired activity level is provided by the doctor taking into account the specifics of the patient.

Both the PALP and the PTAP model are realized as shallow neural networks with one input layer, one hidden layer and an output layer, as depicted in Figure 3. The PALP model takes as input all demographic, intentional and habitual features, and outputs a number corresponding to the expected physical activity level of the patient. The PTAP model uses as input all demographic, intentional and habitual features and the actual and desired physical activity levels. While the latter one is provided by the doctor, the actual physical activity level for the patient is calculated using the HTB app data that objectively monitored travel behaviour and the formula above. The output of the PTAP model is a number between zero and one expressing the degree to which the patient is expected to adhere to the prescribed 
therapy.

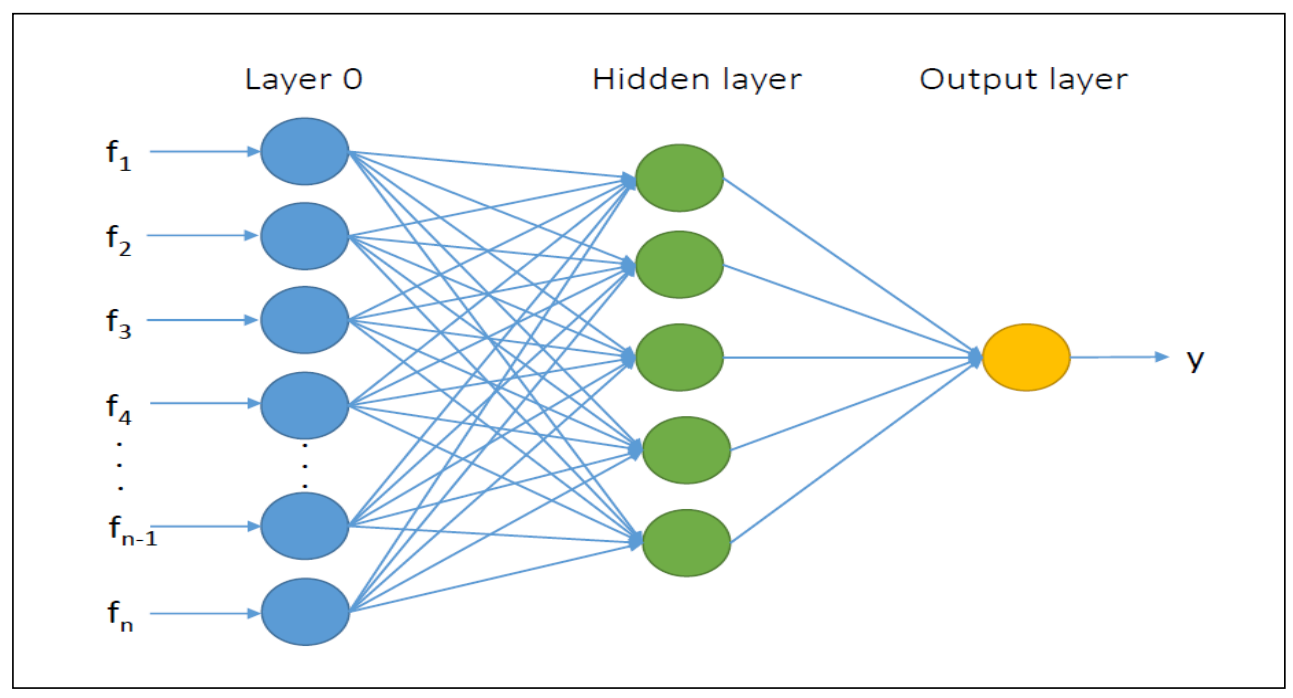

Fig. 5 CTASS prediction model

\subsection{Data analysis and visualization}

Travel data is aggregated in the form of socio-demographic subgroups. These subgroups constitute personalised heuristics, taking into account the behavioural profiles based on the recorded travel behaviour. The aggregated health parameters of the initial report include the total number of trips, trips by each mode, trips by the range of their physical intensity, distance covered by each mode and intensity, corresponding Metabolic Equivalents (METs) (Compendium, 2011) and the duration spent on each trip on daily and weekly basis. This information can help inform the patient's daily and weekly physical activity during the travel activities.

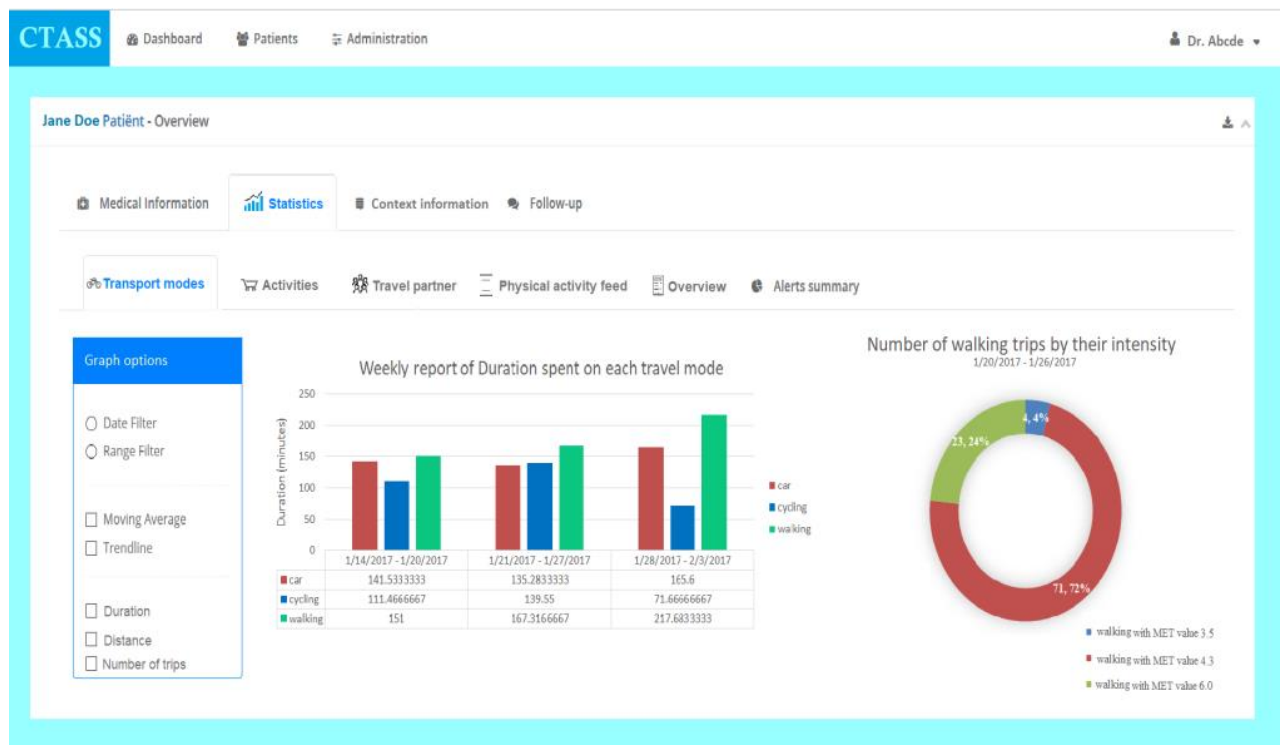

Fig. 6 CTASS interactive dashboard for doctors

After processing the patient's information, the doctor is able to look at the reports on the web-based visualizing 
tool. However, the patients get their personalized reports on their HTB app. Afterwards, in an interactive session, the patient and the doctor will together discuss and agree upon changes in the patient's travel behaviour to be more active. Patients will then be advised to monitor their schedules again in the second period of the study and the CTASS will inform the doctor about (non-)adherence to the revised active travel behaviour therapy. Furthermore, the framework will continue learning the information about underlying travel behaviour parameters (six dimensions) and contextual factors, which will result into the automatic generation of advice. This will identify automatically the specific trips with their properties and recommend the potential substitution of transport modes (from car to bike) for trips based on doctor advice and contextual heuristics. As is shown in Figure 6, the doctor can consult and manage the patient information in the web-based dashboard. Data can be visualized in different kinds of graphs, and filtered over type of data, date and range.

\section{Conclusions and future work}

In this research, we propose a digital framework (CTASS) for personalized travel behaviour advice to cardiac patients. The patient's travel behaviour is monitored by the apps, objectifying the activity of a patient. The behaviour is analyzed semi-automatically by means of a smart decision support system that helps doctors in providing a treatment that is personalized to the specific contexts (e.g. daily activity routine and demographic characteristics) of the patient. In this way, travel related physical activity will be optimized and non-adherence to therapy can be detected. In addition, machine learning models are capable of 1) predicting the likely physical activity level of a patient based on the questionnaires answers, and 2) predicting the likelihood that a patient adheres to a prescribed therapy, based on the questionnaires answers and HTB data.

The system offers only advice about the physical activity level of patients during their trips, but its architecture is generic and pluggable. Information from other external sensors and medical apps can be plugged into the system in order to extend the patient monitoring to cover additional relevant risk factors. For instance, information from a heart rate monitor can also be integrated in the framework, to continuously compare the evolution in cardiac health through an increase in active travel activities and other fitness activities during CR programs.

A group of patients is currently being gathered to start conducting the study. With the CTASS architecture in place, our first research challenge will be to investigate the combined effect of travel behaviour determinants on health parameters (physical activity). In the second phase, we will try to optimize the physical activity generated by travel but including underlying contextual factors of patient profiles (demographics, household interactions, daily utilities). A patient-doctor feedback mechanism through the interactive CTASS framework will effectively incorporate contextual changes to the proposed therapy. Once the patient-tailored feedback is given, patients will be monitored again to evaluate the effectiveness of the personalized therapeutic advice on their physical activity level. As a future optimization we could also cache ids of smartphones of known travel partners, such that they are filled in automatically in the annotated travel diary.

\section{References}

Amiribesheli M, Benmansour A, Bouchachia A (2015) A review of smart homes in healthcare. J Ambient Intell Humaniz Comput 6:495-517. doi: 10.1007/s12652-015-0270-2

Berger M, Platzer M (2015) Field Evaluation of the Smartphone-based Travel Behaviour Data Collection App "SmartMo." Transp Res Procedia 11:263-279. doi: 10.1016/j.trpro.2015.12.023

Bouchard C, Blair SN, Haskell W (2012) Physical Activity and Health-2nd Edition. Human Kinetics

Calvaresi D, Cesarini D, Sernani P, et al (2017) Exploring the ambient assisted living domain: a systematic review. J Ambient Intell Humaniz Comput 8:239-257. doi: 10.1007/s12652-016-0374-3

Chaddha A, Jackson EA, Richardson CR, Franklin BA (2017) Technology to Help Promote Physical Activity. Am J Cardiol New York 119:149-152. doi: http://dx.doi.org/10.1016/j.amjcard.2016.09.025

Cleland CL, Hunter RF, Kee F, et al (2014) Validity of the Global Physical Activity Questionnaire (GPAQ) in assessing levels and change in moderate-vigorous physical activity and sedentary behaviour. BMC Public Heal London 14:. doi: http://dx.doi.org/10.1186/1471-2458-14-1255

Danner UN, Aarts H, Vries NK (2008) Habit vs. intention in the prediction of future behaviour: The role of frequency, context stability and mental accessibility of past behaviour. $\mathrm{Br} \mathrm{J}$ Soc Psychol 47:245-265. doi: 10.1348/014466607X230876

de Nazelle A, Nieuwenhuijsen MJ, Anto JM, et al (2011) Improving health through policies that promote active travel: 
A review of evidence to support integrated health impact assessment

De Vos C, Li X, Van Vlaenderen I, et al (2013) Participating or not in a cardiac rehabilitation programme: factors influencing a patient's decision. Eur J Prev Cardiol 20:341-348. doi: 10.1177/2047487312437057

Francis AJJ, Eccles MPM, Johnston M, et al (2004) CONSTRUCTING QUESTIONNAIRES BASED ON THE THEORY OF PLANNED BEHAVIOUR A MANUAL for HEALTH SERVICES RESEARCHERS

Gerrior S, Juan W, Basiotis P (2006) An easy approach to calculating estimated energy requirements. Prev Chronic Dis 3:A129

Geurs KT, Thomas T, Bijlsma M, Douhou S (2015) Automatic Trip and Mode Detection with Move Smarter: First Results from the Dutch Mobile Mobility Panel. Transp Res Procedia 11:247-262. doi: 10.1016/j.trpro.2015.12.022

Hagströmer M, Oja P, Sjöström M (2006) The International Physical Activity Questionnaire (IPAQ): a study of concurrent and construct validity. Public Heal Nutr Cambridge 9:755-62. doi: http://dx.doi.org/10.1079/PHN2005898

Ham N, Dirin A, Laine TH (2016) Machine learning and dynamic user interfaces in a context aware nurse application environment. J Ambient Intell Humaniz Comput 1-13. doi: 10.1007/s12652-016-0384-1

Kroesen M (2015) Do partners influence each other's travel patterns? A new approach to study the role of social norms. Transp Res Part A Policy Pract 78:489-505. doi: 10.1016/j.tra.2015.06.015

Lee I-M, Shiroma EJ (2014) Using accelerometers to measure physical activity in large-scale epidemiological studies: issues and challenges. Br J Sport Med London 48:197. doi: http://dx.doi.org/10.1136/bjsports-2013-093154

Neven A (2015) Explaining activity-related travel behaviour in persons with disabilities by means of health condition and contextual factors

Newman P, Matan A (2012) Human mobility and human health. Curr Opin Environ Sustain 4:420-426. doi: $10.1016 / j$.cosust.2012.08.005

Nguyen HH, Silva JN (2016) Use of smartphone technology in cardiology. Trends Cardiovasc Med Philadelphia 26:376-386. doi: http://dx.doi.org/10.1016/j.tcm.2015.11.002

Park LG, Beatty A, Stafford Z, Whooley MA (2016) Mobile Phone Interventions for the Secondary Prevention of Cardiovascular Disease. Prog Cardiovasc Dis 58:639-650. doi: 10.1016/j.pcad.2016.03.002

Pr C, Da B, Ra F, et al (1997) Measuring general levels of physical activity: preliminary evidence for the Physical Activity Questionnaire for Older Children. Med Sci Sports Exerc 29:1344-1349. doi: 10.1097/00005768199710000-00011

Rehabilitation AA of C\& P (2004) Guidelines for Cardiac Rehabilitation and Secondary Prevention Programs. Human Kinetics

Safi H, Assemi B, Mesbah M, Ferreira L (2017) An empirical comparison of four technology-mediated travel survey methods. J Traffic Transp Eng (English Ed 4:80-87. doi: 10.1016/j.jtte.2015.12.003

Thompson RG, Kayak H (2011) Estimating Personal Physical Activity from Transport. Australas Transp Res Forum $1-16$

UHasselt I (2012) Handleiding SPARROW app

Vassilaki M, Linardakis M, Polk DM, Philalithis A (2015) The burden of behavioral risk factors for cardiovascular disease in Europe. A significant prevention deficit. Prev Med (Baltim) 81:326-332. doi: 10.1016/j.ypmed.2015.09.024

Widmer RJ, Collins NM, Collins CS, et al (2015) Digital Health Interventions for the Prevention of Cardiovascular Disease: A Systematic Review and Meta-analysis. Mayo Clin Proceedings; Rochester 90:469-480

Zhao F, Ghorpade A, Pereira FC, et al (2015) Stop Detection in Smartphone-based Travel Surveys. Transp Res Procedia 11:218-226. doi: 10.1016/j.trpro.2015.12.019

(2016a) WHO | Cardiovascular diseases (CVDs). In: WHO

(2016b) WHO | Global Status Report on NCDs. In: WHO

(2009) NHTS Documentation. http://nhts.ornl.gov/documentation.shtml. Accessed 31 Aug 2017 